MS19 Materials on energy saving and climate sustainability

Chairs: Dr. Klaudia Hradil, Dr. Claudia Weidenthaler

MS19-01

\section{Carbon dioxide fixation in succulent plants: a crystal engineering, solid state modeling and kinetics approach}

Ebrahiem Botha ${ }^{1}$, Andreas Roodt $^{1}$

1. Chemistry, University of the Free State, Bloemfontein, South Africa

email: ebotha@ufs.ac.za

Photosynthesis in plants is the production of sugar-like entities utilizing carbon dioxide fixation. The equivalent amount of oxygen is produced as 'by-product'. For this conversion to be effective, the plant uses different mechanisms for $\mathrm{CO}_{2}$ fixation. These mechanisms rely on direct access to the Calvin cycle during daytime, where different forms of pyruvate/pyruvic acid are utilized to produce the sugars. Alternatively, indirect access to the Calvin cycle via the preceding Crassulacean Acid Metabolism (CAM) process, may proceed under special conditions of plant stress, or in general in typical Succulent plant families. Storage of the carbon dioxide in different forms of malate/malic acid involves the absorption of the $\mathrm{CO}_{2}$ (often/ principally at night when the stomata open), and then availing it during the next light absorption sequence (closed stomata to preserve moisture) to enter the Calvin cycle. In this project, we aim to fundamentally investigate the physical and chemical processes of carbon dioxide fixation. This we do by using artificial model 'plant leaf analogs', by crystal engineering of appropriate active systems. Therefore, further enabling the utilization of the carbon dioxide as value-added building block for synthetic methodologies via biomimetic strategies.

A series of rhodium and other platinum group metal complexes, including carboxylates were synthesized to mimic the active systems in the plant leaves that were probed using a plant sample box we custom-built for the project. Some rhodium model systems, wherein different non-labile bidentate ligand systems with different stereo-electronic properties have been included, were evaluated kinetically. The complexes were characterized using NMR, FTIR, UV/ vis and SC-XRD. These complexes include simple square planar geometries with labile ligand systems to mimic (reactive) carbon dioxide models. Preliminary kinetics were studied to evaluate both steric and electronic effect at these metal centers.

Selected complexes were also studied by single crystal X-ray crystallography to verify the bonding modes of the bidentate ligands therein, as well as the carbon dioxide and model ligands' coordination geometries.

Selected details of these compounds will be discussed in this presentation as well as some future strategies to be followed.
References:

[1] Smith, G. et al. Field Guide to Succulents of Southern Africa, Struik Nature, 2017, ISBN: 177584367X, 9781775843672

[2] Warsink, S. et al, European Journal of Inorganic Chemistry. 2018. DOI: 10.1002/ejic.201800293.

[3] Otto, S. et al, Croatica Chemica Acta, Submitted

Keywords: Succulents, Crystallography, Kinetics 